\title{
Prognosis genes in gastric adenocarcinoma identified by cross talk genes in disease-related pathways
}

\author{
LIZHI ZHAO ${ }^{1}$, HAICHUN LEI ${ }^{1}$, LI SHEN $^{1}$, JIQUAN TANG ${ }^{1}$, ZHIWEI WANG $^{1}$, \\ WEISONG BAI ${ }^{1}$, FENG ZHANG ${ }^{1}$, SHOULI WANG ${ }^{1}$ and WEIHUA LI $^{2}$ \\ ${ }^{1}$ Department of Digestive Surgery, Hanzhong Central Hospital, Hanzhong, Shaanxi 723000; \\ ${ }^{2}$ Center Lab, The People's Hospital of Gansu, Lanzhou, Gansu 730000, P.R. China
}

Received April 11, 2016; Accepted March 14, 2017

DOI: $10.3892 / \mathrm{mmr} .2017 .6699$

\begin{abstract}
The aim of the present study was to investigate the prognostic value of genes that participate in the development of gastric adenocarcinoma, via exploring gene cross talk in disease-related pathways. Differentially expressed genes (DEGs) in the gastric samples were identified by analyzing the expression data downloaded from the GEO database. The DEGs were subjected to the human protein-protein interaction (PPI) network to construct the PPI network of DEGs, which was then used for the identification of key genes in cancer samples via the expression deviation score and degree in the network. A total of 635 DEGs, including 432 downregulated and 203 upregulated ones were screened in the gastric adenocarcinomas samples. The PPI network of DEGs comprised 590 DEGs and 4,299 interaction pairs. A total of 200 key genes were obtained, which were significantly enriched in six downregulated and six upregulated pathways. Cross talk genes in the connected pathways were analyzed, and the Kyoto Encyclopedia of Genes and Genomes pathways hsa00980 (Metabolism of xenobiotics by cytochrome P450) and hsa00982 (Drug metabolism) were reported to share 8 cross talk genes: ADH7, ALDH3A1, GSTA1, GSTA2, UGT2B17, UGT2B10, ADH1B and CYP2C18. Among all cross talk genes, ADH7, ALDH3A1 and CLDN3 were the most specific genes. The high- and low-risk samples identified by the prognosis model presented a remarkable difference in total survival time, indicating its robustness and sensitivity as the prognosis genes for gastric adenocarcinoma. ADH7, ALDH3A1, GSTA1, GSTA2, UGT2B17, UGT2B10, ADH1B, CYP2C18ADH7, ALDH3A1 and CLDN3 may be used as the prognosis markers and target biomarkers for chemotherapies in gastric adenocarcinoma.
\end{abstract}

Correspondence to: Dr Weihua Li, Center Lab, The People's Hospital of Gansu, 204 West Donggang Rood, Lanzhou, Gansu 730000, P.R. China

E-mail: liweiweiijjj@hotmail.com

Key words: gastric adenocarcinomas, cross talk gene, protein-protein interaction, pathway, prognosis

\section{Introduction}

Gastric cancer affects $\sim 1$ million people every year, and $70-85 \%$ of them will die within 5 years following diagnosis (1). Gastric adenocarcinoma is one of the leading causes of cancer related death worldwide, even though there is a rapid development of medical technology, with a higher incidence in Asian countries, including China (2). A high-salt diet, infectious agents and smoking are the environmental risk factors for gastric adenocarcinoma (3). Although resection could be curative, the prognosis of gastric adenocarcinoma patients at advanced stage is still very poor following radical resection and surgical treatment $(4,5)$. Therefore, it is urgently needed to identify the prognostic and predictive biomarkers or models to develop the most effect methods to improve the clinical outcome in gastric adenocarcinoma.

The contribution of genetic alterations to the initiation and development of gastric cancer have been reported in many studies. For example, TGR5 is overexpressed in most gastric intestinal-type adenocarcinomas (6). The upregulation of CD24 is correlated with venous invasion, lymphatic invasion and lymph node metastasis of gastric carcinoma (7). Expression levels of $\mathrm{ABCB} 1, \mathrm{ABCG} 2$ and $\mathrm{CD} 133$ are correlated with the differentiation degree of gastric cancer (8). The connections between genetic variation and the prognosis status of gastric adenocarcinoma patients have also been explored, and the overexpression of HER2 and EGFR were identified as the prognostic factors in gastric cancer (9). HER3 expression was associated with the decreased survival of gastric cancer, acting as a prognosis factor for patients at the advanced stage (10). Tumoral FOXP3 expression in gastric adenocarcinomas is reported to be related with the favorable clinicopathological variables (11). Although these studies have identified some prognosis genes in stomach cancer, the demand for more prognostic biomarkers remains to be met for developing targeted therapies.

During the development of cancers, certain genetic changes will lead to the functional abnormality of related pathways, which may influence other pathways and result in a progression of cancer. Cross talk genes refer to the genes that connecting two or more pathways, in which the abnormality of one pathway can be passed to another because of these genes. The cross talk between EPAS-1/HIF-2 $\alpha$ and the PXR signaling pathway were 
reported as the regulatory factor for multi-drug resistance of stomach cancer cell (12). The SKP2 gene was demonstrated to regulate cancer progression by participating in the cross talk with other major cancer signaling pathways $(13,14)$. Moreover, the STAT3 gene is reported to interact with the SKP2/p27/p21 pathway to regulate the invasion and motility of gastric cancer cells (15).

To explore potential novel biomarkers for the treatment and prognosis of gastric adenocarcinoma, microarray data of gastric adenocarcinomas were screened and downloaded from GEO and TCGA databases, and the cross talk between genes was analyzed in disease-related pathways.

\section{Materials and methods}

Microarray data. Gene expression microarray data (GSE13861) were downloaded from GEO (Gene Expression Omnibus) database (https://www.ncbi.nlm.nih.gov/geo/), including 65 primary gastric adenocarcinomas samples, 16 interstitial gastric adenocarcinoma samples and 19 surrounding normal fresh frozen tissues. Gene annotation data were also downloaded from Illumina HumanWG-6 v3.0 expression beadchip (https://www.ncbi.nlm.nih.gov/geo/query/ acc.cgi?acc=GPL6884). Only gastric adenocarcinomas samples and surrounding normal samples were analyzed in following analysis.

Data normalization. Microarray data were transformed into gene symbols, and the average expression values were used as the expression levels of genes. The Z-score method (16) was applied for data normalization and the expression variations of genes in cancer samples were also extended by this method.

Differentially expressed genes screening. The BioConductor version 1.6 (R-2.1) Limma package (17) (http://www. bioconductor.org/packages/release/bioc/html/limma.html) was utilized for the screening of differentially expressed genes (DEGs) between gastric adenocarcinomas samples and surrounding normal samples. False discovery rate (FDR) $<0.01$ and $\mid \operatorname{logfcl}>1.2$ were the cut-off criteria.

Protein-protein interaction network. The protein-protein interaction (PPI) pairs were downloaded from BioGrid (http://thebiogrid.org/) and HPRD (http://www.hprd.org/) database, which were then overlapped to obtain the whole human PPI network. Next, the proteins, which are correlated with at least 3 DEGs in the human PPI network were screened, and these proteins and their interactions were visualized in the PPI network of DEGs.

Topological analysis. The topological properties of both the human andDEGPPInetwork were analyzed using the Cytoscape software version 3.4.0 (http://www.cytoscape.org/) (18), including nodes' degree, average shortest path, network centrality, eccentricity and topological factors. The topological property differences between these two PPI network were compared.

Key gene selection in PPI interaction network. Key genes among the DEGs were selected according to the deviation score and degree in the PPI network. To calculate the deviation score, the expression interval I (average expression value + standard deviation (SD), average expression value-SD) of each gene was firstly defined according to their expressions in normal samples. If the expression of a gene is beyond the interval of I, this gene will be considered to be key genes involved in gastric adenocarcinomas. The extra expression value of the gene will be next used for the calculation of deviation score. The formula for the deviation computing is listed as below:

$$
\begin{aligned}
& \mathrm{W}=\text { score } \times \text { degre } \epsilon \\
& \text { score }=\sqrt{\sum_{1}^{\mathrm{n}}\left(\mathrm{d}_{\mathrm{i}}-\mathrm{d}\right)^{2}}
\end{aligned}
$$

$d_{i}$ represents the gene expression value of sample $i$, and, if $d_{i}$ is larger than average expression value $+\mathrm{SD}$, then the value of $d$ will be recorded as average expression value $+S D$; if $d_{i}$ is lower than average expression value-SD, then, the value of $\mathrm{d}$ will be recorded as average expression value-SD. The score was normalized to the range of $0-10$ by using distance. Degree was normalized by using the $\log 2$ value. Finally, W was calculated to get the rank of genes.

Genes with higher deviation score and node degree in the PPI network have much more important roles in the gastric adenocarcinoma samples, and they will be recognized as the key genes. In this analysis, the genes with $\mathrm{W}$ rank of the top 100 and last 100 will be selected at the key genes.

Pathway enrichment and hierarchical clustering. The key genes with the top $50 \mathrm{~W}$ scores were conducted KEGG pathway enrichment using DAVID (https://david.ncifcrf. gov/) (19). The KEGG pathway is a collection of manually drawn pathway maps representing data regarding the molecular interaction and reaction networks, including metabolism, genetic information processing, environmental information processing, cellular processes, organismal systems, human diseases and drug development (www. genome.jp/kegg/pathway.html). The FISHER hypergeometric distribution test (20) was applied, $\mathrm{P}<0.05$ was the threshold. The significant pathways were then performed hierarchical clustering analysis, the changes of pathways (the pathscore) in every sample was presented by gene expression in the pathways. The pathscore was computed using the following formula.

$$
\text { pathscore }=\log \frac{\sqrt{\sum_{i}^{m} \omega\left(d_{i}-\overline{d_{i}}\right)^{2}}}{\sqrt{\sum_{j}^{n} \omega\left(d_{j}-\overline{d_{j}}\right)^{2}}}
$$

$m$ represents the upregulated genes and $n$ represents the downregulated genes in pathway $\mathrm{p}$, while represents the average expression level of upregulated gene i or down-regulated gene $\mathrm{j}$ in normal samples. The deviation of pathway in cancer samples was calculated using Euclidean Distance, pathscore is the log value of pathway deviation. Pathscores larger than 0 represent the upregulation of the pathway, while the lower than 0 represents the downregulation of the pathway. The hierarchical clustering of pathways was conducted using the correlation center method, as previously described (21). 
Table I. Topological properties comparison of DEG PPI network and human PPI net-work.

\begin{tabular}{lcc}
\hline Features & $\begin{array}{c}\text { DEG PPI } \\
\text { network }\end{array}$ & $\begin{array}{c}\text { Human PPI } \\
\text { network }\end{array}$ \\
\hline Degree & 3.8600 & 7.0100 \\
Eccentricity & 5.4300 & 6.5100 \\
Average shortest path length & 3.2300 & 2.970 \\
Closeness centrality & 0.3600 & 0.3500 \\
Topological coefficient & 0.2400 & 0.1700 \\
Betweenness centrality & 0.0038 & 0.0049 \\
\hline
\end{tabular}

DEG, differentially expressed genes; PPI, protein-protein interaction.

Table III. Support vector machine model report.

\begin{tabular}{lccc}
\hline & Precision & Recall & F1-score \\
\hline Cancer sample & 0.97 & 0.94 & 0.95 \\
Normal samples & 0.82 & 0.9 & 0.86 \\
Average/total & 0.93 & 0.93 & 0.93 \\
\hline
\end{tabular}

Pathway correlation analysis and identification of cross talk genes. The correlations between pathways were calculated using the Spearman's rank correlation analysis, and the pathways that were positively connected and negatively connected were collected separately. Following that, the cross talk genes between connected pathways were identified.

Prognosis gene screening. The cross talk genes among connected pathways were used for the construction of prognosis model via the Random Forests algorithm. For the testing of the prognosis model, all samples were divided into 10 parts, in which nine parts of samples were used as the training set, and the left one part of samples was used for the validation set of the model. After 10 times' testing and validation, the ROC (receiver operating characteristic) curve was drawn to evaluate the ability of classification and the robust of the prognosis model. The precision, recall rate and F1-score were calculated to assessed the accuracy of the model. The F1 score was computed using the following formula: $F 1=2 x$ (precision $\mathrm{x}$ recall $) /($ precision + recall).

Prognosis gene validation in TCGA database. The expression profiles of 287 gastric adenocarcinoma samples, along with the clinical data were downloaded from the TCGA database (tcga-data.nci.nih.gov) for the validation of the prognosis model. The samples were considered as high risk if at least one cross talk gene was differentially expressed, whereas the samples with no differentially expressed cross talk genes were considered as low risk. Finally, the robustness and sensitivity of the cross talk genes were tested by comparing the survival curves of the high-risk and low-risk gastric adenocarcinoma samples.
Table II. Connected pathway numbers of the cross talk genes.

\begin{tabular}{lclc}
\hline $\begin{array}{l}\text { Cross talk } \\
\text { gene }\end{array}$ & $\begin{array}{c}\text { Pathway } \\
\text { number }\end{array}$ & $\begin{array}{c}\text { Cross } \\
\text { talk gene }\end{array}$ & $\begin{array}{c}\text { Pathway } \\
\text { number }\end{array}$ \\
\hline GSTA2 & 2 & ADH1B & 3 \\
UGT2B17 & 3 & ALDH3A1 & 2 \\
UGT2B10 & 2 & COL4A1 & 2 \\
ACAT1 & 2 & CLDN3 & 2 \\
CLDN1 & 3 & ADH7 & 4 \\
MCEE & 2 & CLDN7 & 2 \\
E2F3 & 2 & ADH1B CYP2C18 & 3 \\
CLDN4 & 2 & CDK4 & 3 \\
\hline
\end{tabular}

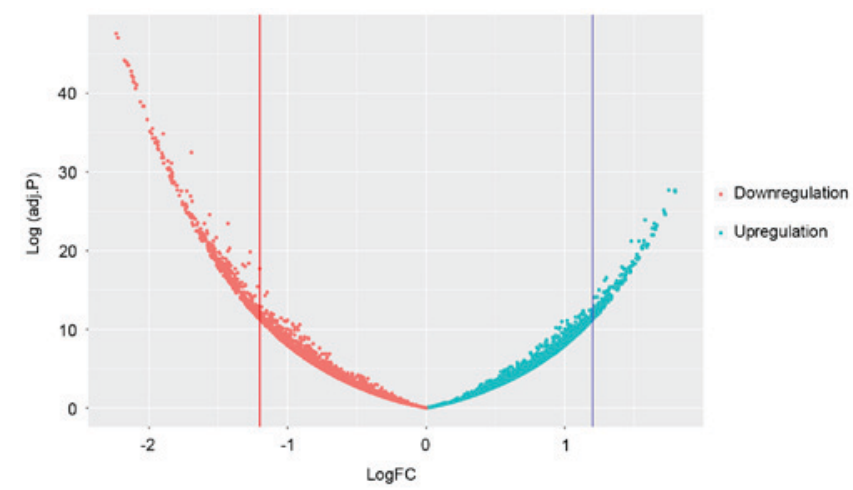

Figure 1. Volcano plot of genes. The volcano plot indicates the magnitude of differential expression between gastric adenocarcinoma samples and surrounding normal samples. The horizontal line marks the threshold $(\mathrm{P}<0.01)$ to define one gene as upregulated (red) or downregulated (blue), combining the change $>2$ fold.

\section{Results}

Differentially expressed genes. A total of 635 DEGs, including 432 downregulated and 203 upregulated ones were screened in gastric adenocarcinomas samples comparing to surrounding normal samples. The distribution of P-values and fold change was presented in Fig. 1.

Protein-protein interaction network. The human PPI network was composed of 14,553 proteins and 662,360 interacting pairs, and, in the PPI network of DEGs there were 590 nodes (DEGs) and 4,299 lines (interactions) (Fig. 2).

Topological properties of PPI network. The distribution of node degree in the PPI network is presented in Fig. 3. It was observed that ' 4 ' was the most common node degree. The curve of average shortest path, network centrality, eccentricity and topological coefficient presented $>1$ peak, indicating that there are multiple functional modules in the PPI network.

The topological properties of the human PPI network and DEG PPI network were compared (Table I), it is indicated that DEGs had a lower degree (3.86 vs. 7.01 ), eccentricity (5.43 vs. 6.511) and betweenness centrality (0.0038 vs.0.0049), longer average shortest path length (3.23 vs. 2.97$)$ and topological 


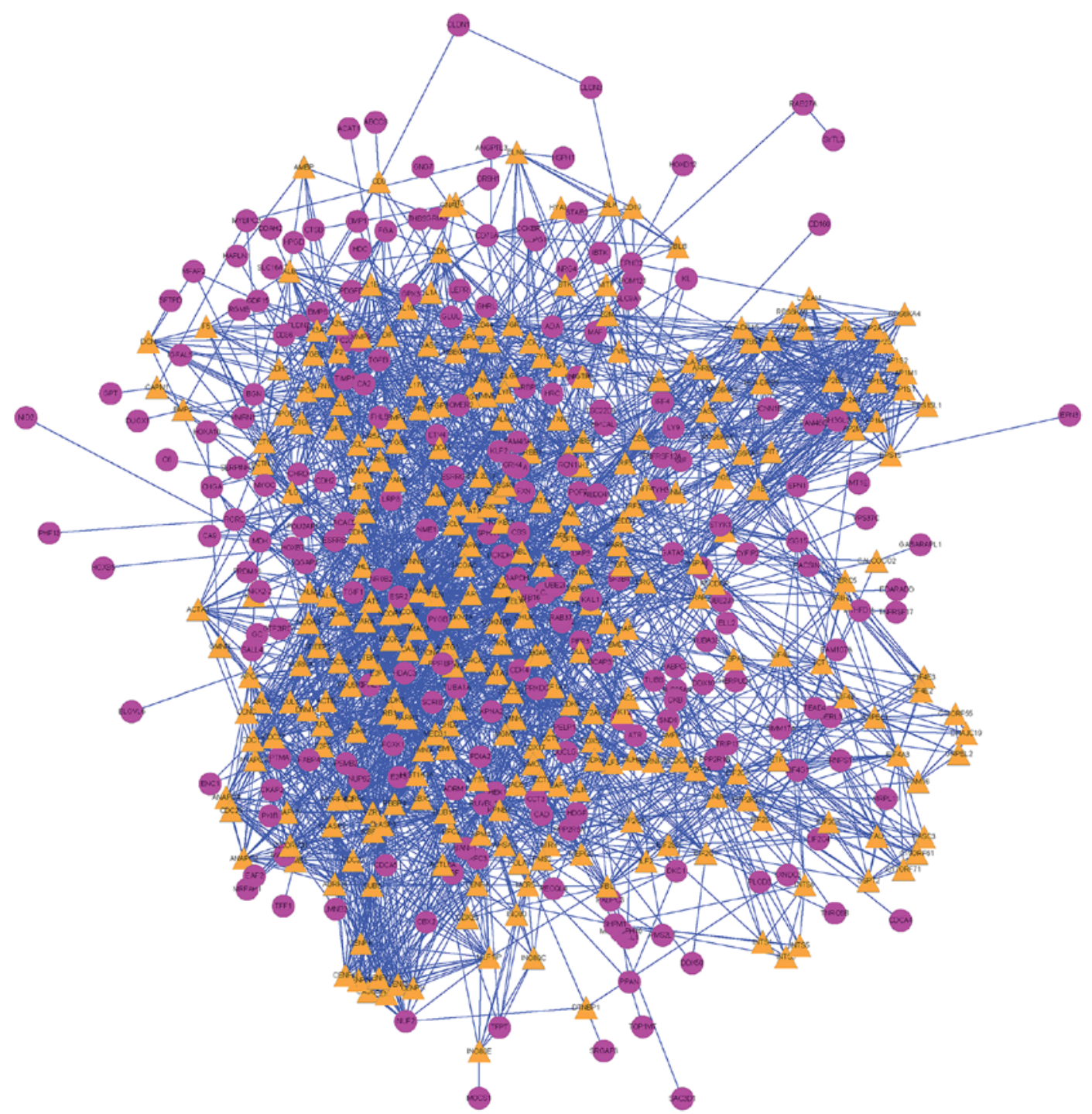

Figure 2. Protein-protein interaction network. Differentially expressed genes are marked in purple, while the expanding genes are marked in orange.
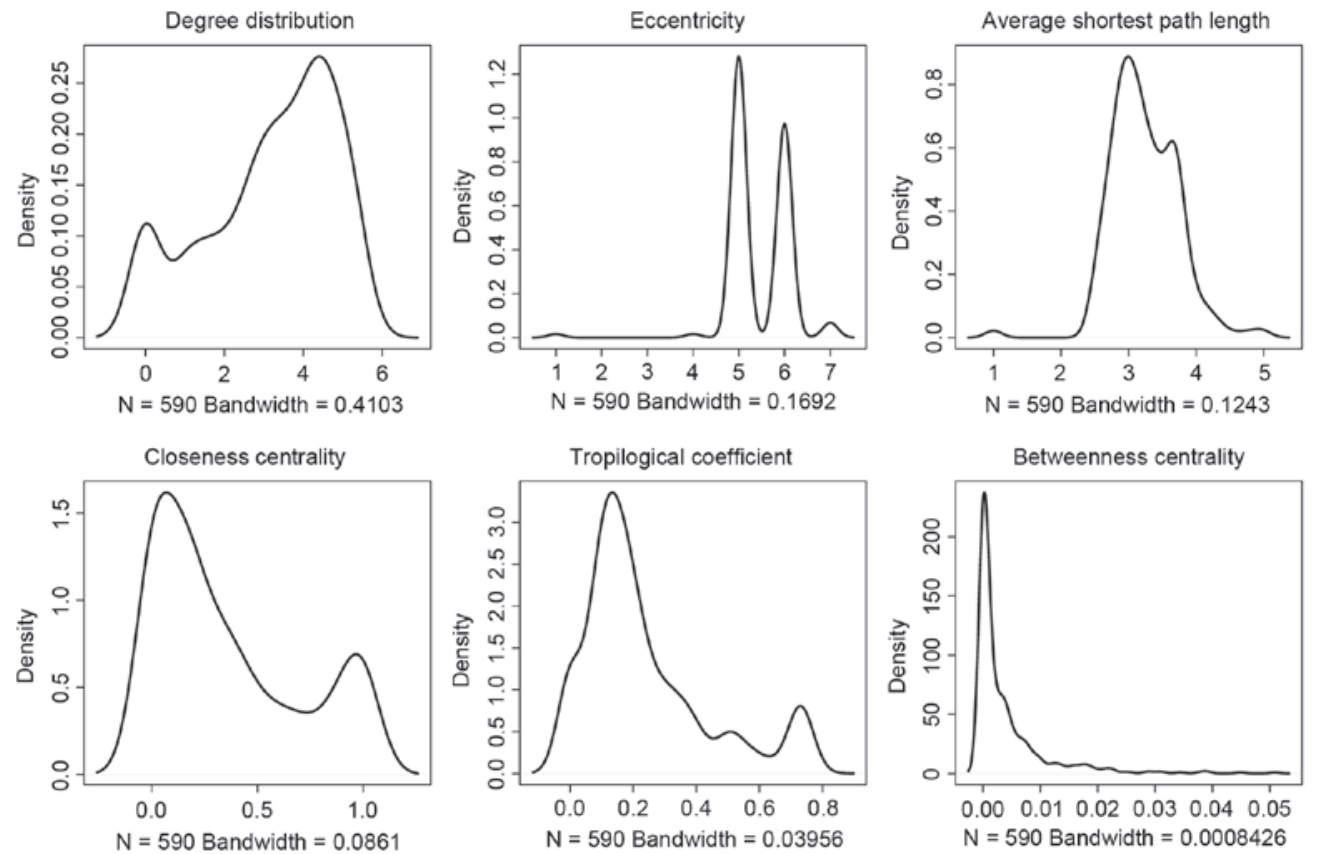

Figure 3. Topological properties of protein-protein interaction network. 


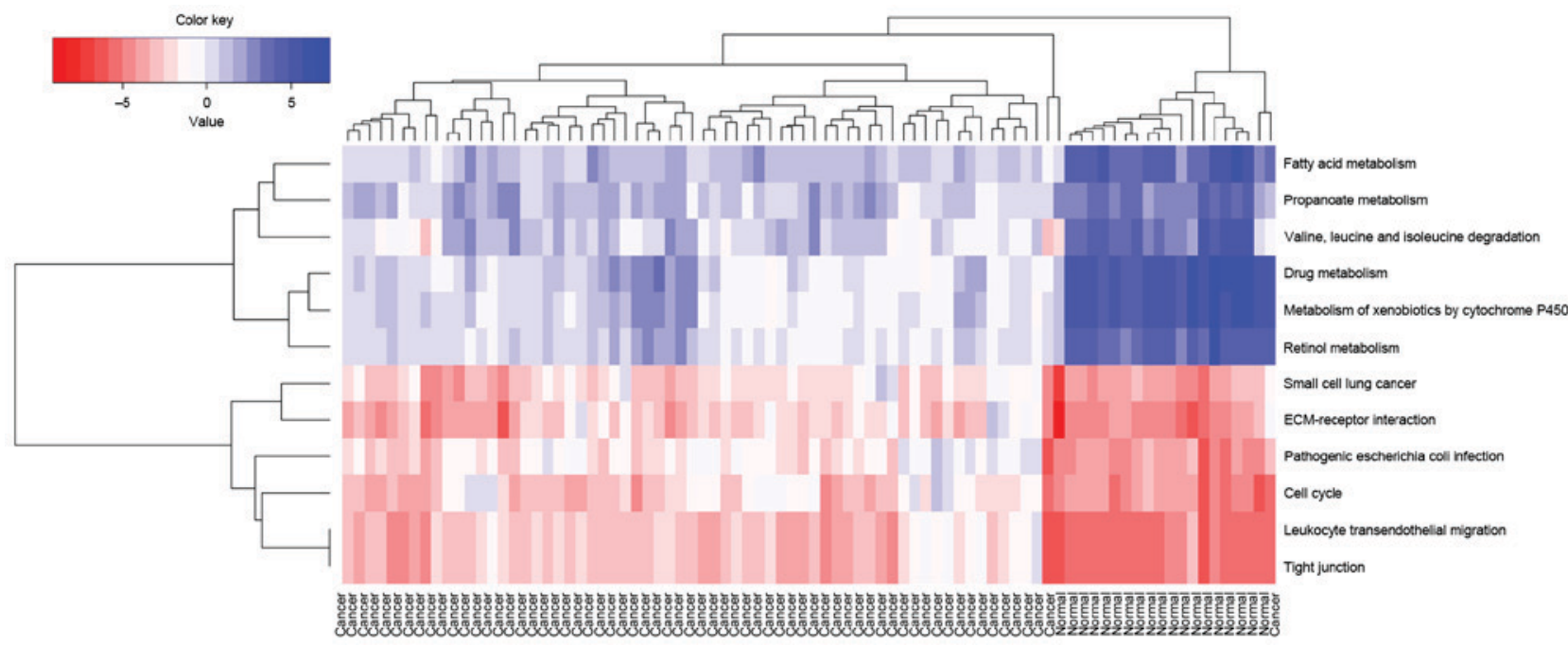

Figure 4. Hierarchical clustering analysis of pathways. All gastric adenocarcinoma samples and normal tissue samples are on the x-axis, while the significantly enriched pathways are on the y-axis. The color changes from red to blue represent the changes from high score to low score of each pathway in the samples.

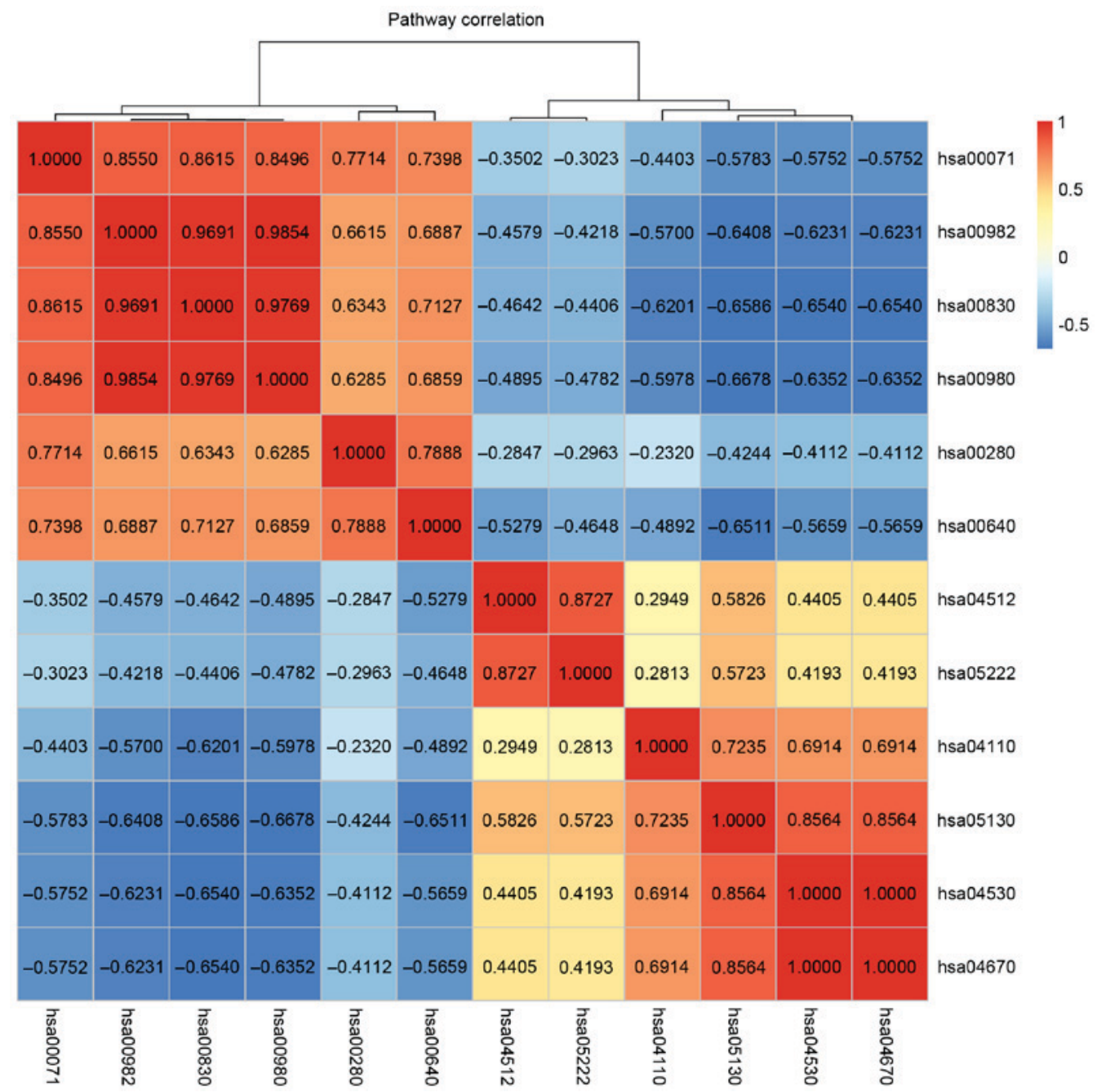

Figure 5. Correlation analysis of functional pathways. The bright yellow/orange represents the positive correlation between pathways, while dark blue represents the negative correlations.

coefficient (0.238 vs. 0.17$)$, as well as equal closeness centrality (0.357 vs. 0.35$)$ compared to the normal human genes. All these changes suggested that in the PPI network, i) the contribution of genes became lower; ii) the specificity of the network was increased; iii) the signal conducting power among genes became weak. 


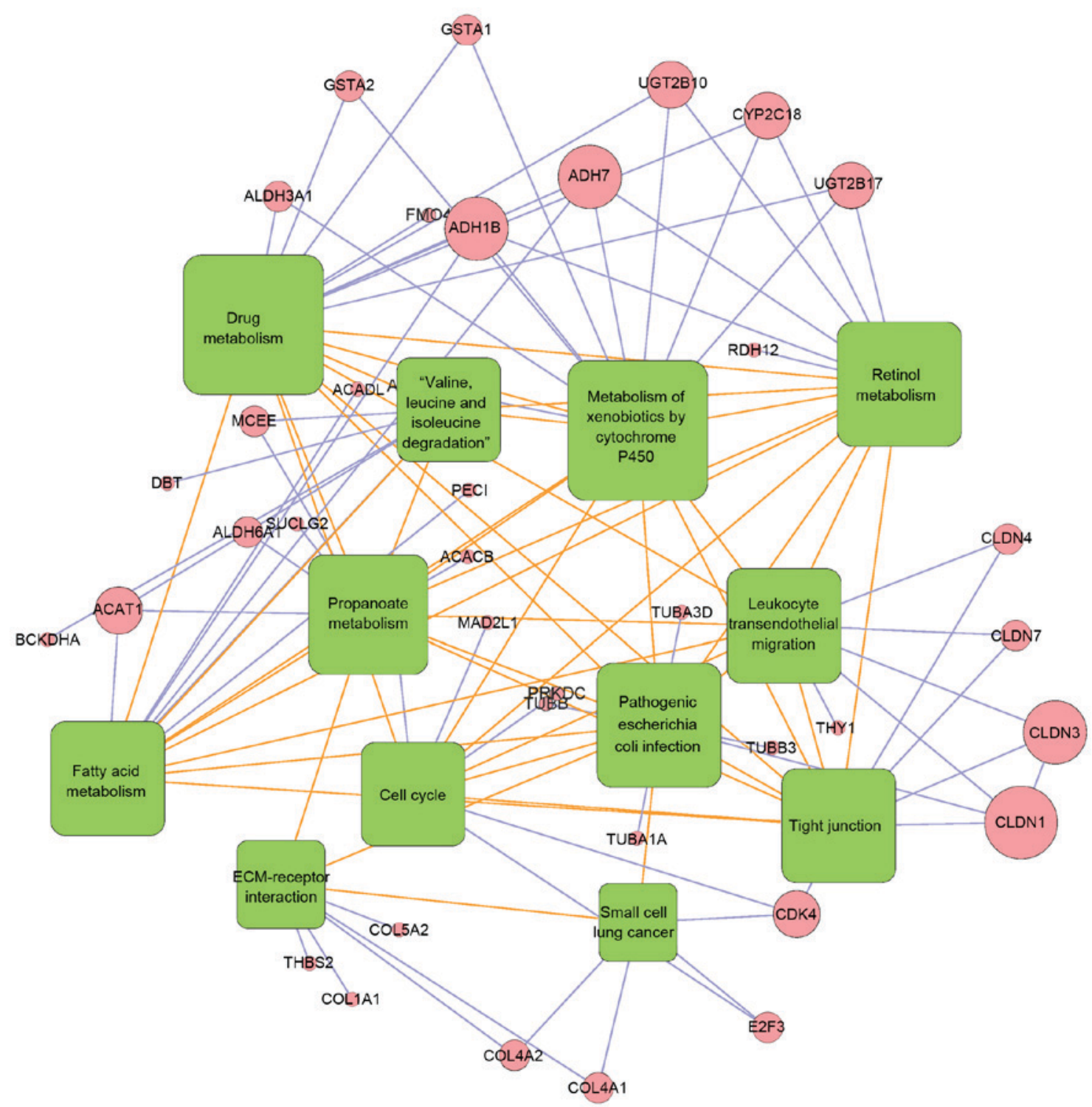

Figure 6. Pathway-pathway connection network. Pathways are shown in green boxes, genes are in pink circles. The connected pathways of differentially expressed genes are linked by blue lines, while the correlated pathways are linked by orange lines. The size of each hub pathways/genes are the representatives of their degrees in the network.

Key genes and the enriched pathways. In all, by calculating the deviation score and the $\log 2$ transformation of the degree, 200 key genes were obtained. These genes had significant expression deviation from normal samples, while possessed higher degrees in the PPI network.

Pathway enrichment analysis reported six upregulated pathways and six downregulated pathways, which had no crossovers. The significant pathways were the cancer, cell cycle, cell apoptosis, immunity and metabolism-related pathways, suggesting the underlying mechanism of the initiation and progression of gastric adenocarcinoma. The disorder of cell cycle may contribute the development of cancer and the abnormality of metabolism may be involved in the metastasis of cancer cells, disease recurrence and apoptosis escape.

Hierarchy analysis discovered that the pathscore of the 12 significant pathways could distinguish cancer samples from normal samples. In addition, it is observed that the deviations of each pathways in cancer samples and normal samples shared similar changing trends (Fig. 4). The correlations between all these 12 pathways were visualized using a heatmap (Fig. 5). The genes existing in $>1$ pathways were the cross talk genes, and the differential expression of these genes imply the abnormality of the related pathways. The complicated connections of pathway-gene and gene-gene are displayed in Fig. 6, which comprised 52 nodes (12 pathways/44 genes) and 111 lines (the relationship). Among their connection pairs, the Kyoto Encyclopedia of Genes and Genomes (KEGG) pathways hsa00980 (Metabolism of xenobiotics by cytochrome P450) and hsa00982 (Drug metabolism) shared eight cross talk genes: ADH7, ALDH3A1, GSTA1, GSTA2, UGT2B17, UGT2B10, $\mathrm{ADH} 1 \mathrm{~B}$ and $\mathrm{CYP} 2 \mathrm{C} 18$. There were 17 genes that are involved in $>1$ pathway, including ADH7 (shared by four pathways); UGT2B17, CLDN1, ADH1B, CYP2C18, and CDK4 (shared by three pathways) (Table II).

Prognosis gene screening. A support vector machine prognosis model was constructed using the cross talk genes, and the ROC curve of five-fold cross validation is presented in 


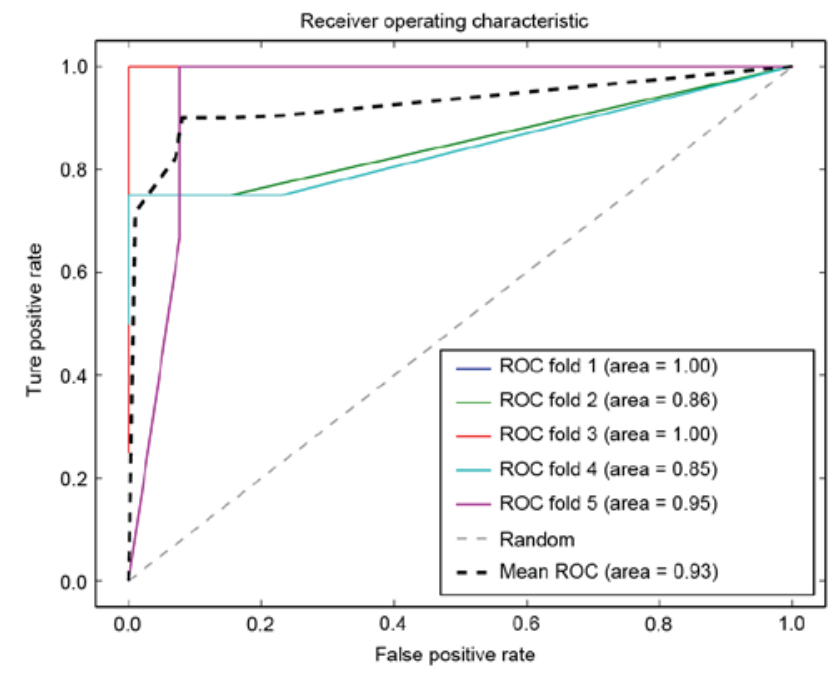

Figure 7. ROC curve using five-fold cross validation. ROC, receiver operating characteristic.

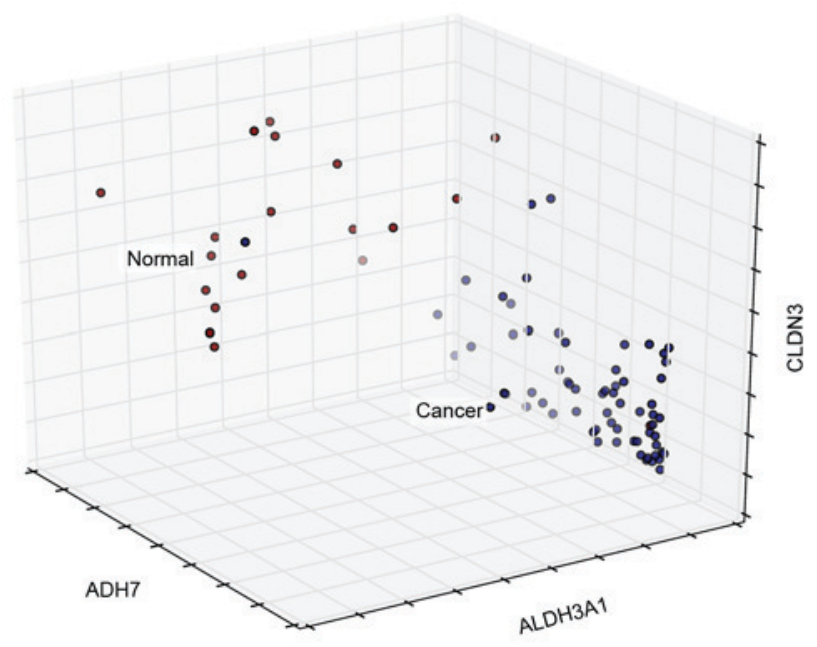

Figure 8. Sample 3D distribution. $\mathrm{X}, \mathrm{Y}$ and $\mathrm{Z}$ axes represent the expression levels of the three genes.

Fig. 7. It was observed that the lowest precision of the prediction was 0.85 , and the average precision was 0.94 , indicating the robustness and precision of the prognosis model. The model also exhibited a high recall rate and Fl-score (Table III).

The classification ability of the cross talk genes to normal and cancer samples was also presented using a $3 \mathrm{D}$ visualization method (Fig. 8). Among all cross talk genes, ADH7, ALDH3A1 and CLDN3 exhibited the most specific classification characteristics.

Moreover, the prognosis model was validated using other independent expression profiles downloaded from TCGA. The samples with at least one differentially expressed cross talk genes were defined as the high-risk samples, while the samples with no differentially expressed cross talk gene were defined as the low-risk samples. The survival curve of the high-risk and low-risk samples is presented in Fig. 9, and the result indicated that these two groups of samples were different significantly in total survival time $(\mathrm{P}=0.03)$, indicating its robustness and sensitivity as the prognosis genes for gastric adenocarcinoma.

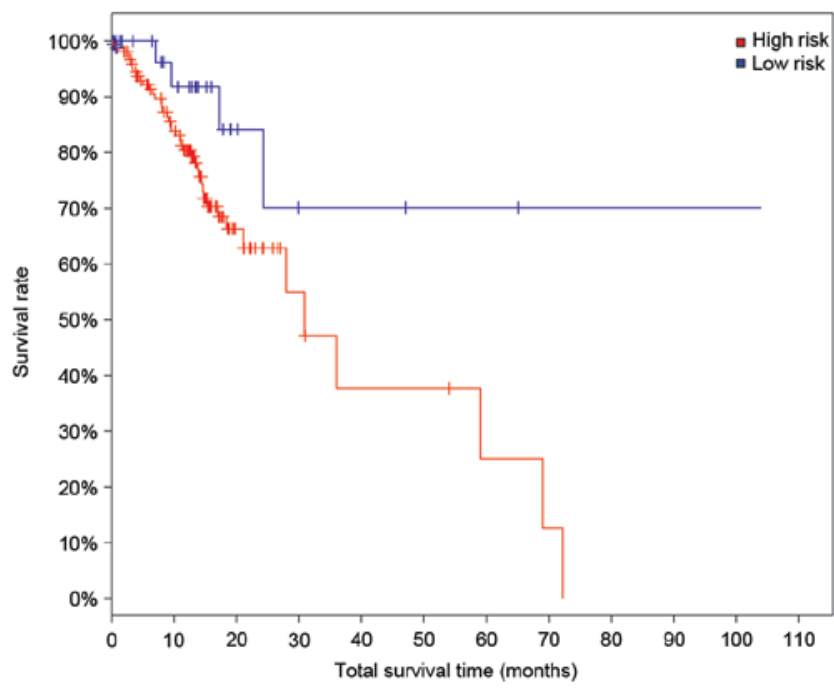

Figure 9. Survival analysis of high- and low-risk group of gastric adenocarcinoma. Log rank test $\mathrm{P}$-value $=0.0300$. High risk group, cases with alteration(s) in query gene(s); Low risk group, cases without alteration(s) in query gene(s).

\section{Discussion}

In the present study, a total of 635 DEGs were screened in the gastric adenocarcinoma samples, including 432 downregulated ones and 203 upregulated ones. The PPI network of DEGs were composed of 590 DEGs and 4,299 interaction pairs. A total of 200 key genes were identified, which were significantly enriched in six downregulated and six upregulated pathways. Cross talk genes in the connected pathways were then analyzed, and KEGG pathways hsa00980 (Metabolism of xenobiotics by cytochrome P450) and hsa00982 (Drug metabolism) shared 8 cross talk genes: ADH7, ALDH3A1, GSTA1, GSTA2, UGT2B17, UGT2B10, ADH1B and CYP2C18. Among all cross talk genes, ADH7, ALDH3A1 and CLDN3 were the most specific. The high- and low-risk samples identified by the prognosis model established by cross talk genes presented a remarkable difference in total survival time, indicating its robustness and sensitivity.

Correlates between expression of various metabolizing enzymes with risk of malignancies have been observed for many years (22). It was reported that the dysfunction of pathways hsa00980 (Metabolism of xenobiotics by cytochrome P450) and hsa00982 (Drug metabolism) would induce the drug resistance or adverse reaction during the chemotherapy for gastric adenocarcinoma by interrupting drug metabolism and promoting drug excretion (23-25). These two pathways were demonstrated shared 8 cross talk genes, and they are the nodes in the pathway-gene network with high degrees. Considering their close connection with other key genes and pathways involved in gastric adenocarcinoma, these genes and pathways may be the potential targets for the treatment. The prognostic roles of these 8 genes for normal samples and gastric cancer samples have been validated in the current study. Of all these genes, ADH7 and ADH1B belong to the alcohol dehydrogenase family, ALDH3A1 is from aldehyde dehydrogenase family, GSTA1 and GSTA2 are from the glutathione transferase family, UGT2B17 and UGT2B10 are from 
the uridine diphosphate-glucuronosyltransferase 2B family, and CYP2C18 is from the cytochrome P-450IIC family. There are evidences proving the connections between these families and gastric adenocarcinomas (26-30).

ADH7 (alcohol dehydrogenase 7), the gene expressed primarily in the upper gastrointestinal tract, is proved to be participated in the metabolism of xenobiotics by cytochrome P450: It is implicated in the metabolism of ethanol occurred in gastroesophageal tissues before the absorption in the blood (31,32). Single nucleotide polymorphisms in ADH7 are reported as a susceptible factor for cancer and drug dependence (33). ALDH (aldehyde dehydrogenase) is the enzyme responsible for the oxidation of acetaldehyde, and it is reported that cancer cells exhibit a much greater capability in ethanol oxidation but less ability for its remove (26). A European study indicated the genetic variants at the loci of ADH1 and ALDH2 may influence GC risk (27). Jelski et al (34) suggested $\mathrm{ADH}$ and ALDH may be used as the candidate tumor markers in pancreatic cancer. The ADH1B*1 allele is proven to be associated with an increased risk of esophageal cancer (35). Besides the involvement in the metabolism of xenobiotics by cytochrome $\mathrm{P} 450$ and drug metabolism pathways, ADH7, ALDH3A1 and ADH1B were also the cross talk genes with the higher connections with pathways, suggesting the possibility for these genes to be used as the biomarkers for the diagnosis and prognosis of gastric adenocarcinoma.

Claudins (CLDNs) are the major tight-junction proteins, which expressed at the apical membrane of epithelial cells. The main function of CLDNs is the control to paracellular permeability and the maintenance of epithelial polarity (36). The expression reduction or loss of CLDNs has been revealed to be able to promote the invasion and metastasis of malignant tumor cells, including the tumor in gastrointestinal tract $(37,38)$. CLDNs are good biomarkers for the determination of the differentiation and aggressiveness of gastric cancer (39). CLDN3 belongs to the CLDNs family, and it is expressed in metaplastic mucosa and gastric carcinomas $(40,41)$. Overexpression of CLDN3 has been recognized as a prognostic indicator for ovarian serous carcinomas (42). Jung et al (43) reported that CLDN3 is the most important indicator for the lymphatic invasion process in gastric cancer. Consistent with previous findings, CLDN3 was identified to be a classification gene for gastric adenocarcinoma in the present study.

The analysis on cross talk genes between dysfunctional pathways is useful in finding the potential biomarkers in cancer. ADH7, ALDH3A1, GSTA1, GSTA2, UGT2B17, UGT2B10, ADH1B, and CYP2C18 and CLDN3 may be used as the prognosis factors and target biomarkers for chemotherapies in gastric adenocarcinoma.

\section{References}

1. Oliveira C, Pinheiro H, Figueiredo J, Seruca R and Carneiro F: Familial gastric cancer: Genetic susceptibility, pathology, and implications for management. Lancet Oncol 16: e60-e70, 2015.

2. Kang W, Tong JH, Lung RW, Dong Y, Zhao J, Liang Q, Zhang L, Pan Y, Yang W and Pang JC, et al: Targeting of yap1 by microrna-15a and microrna-16-1 exerts tumor suppressor function in gastric adenocarcinoma. Mol Cancer 14: 52, 2015.

3. Ferlay J, Shin HR, Bray F, Forman D, Mathers C and Parkin DM: Estimates of worldwide burden of cancer in 2008: Globocan 2008. Int J Cancer 127: 2893-2917, 2010.
4. Lin SJ, Gagnon-Bartsch JA, Tan IB, Earle S, Ruff L, Pettinger K, Ylstra B, van Grieken N, Rha SY, Chung HC, et al: Signatures of tumour immunity distinguish Asian and non-Asian gastric adenocarcinomas. Gut 64: 1721-1731, 2015.

5. Stewart B and Wild CP: World cancer report 2014. World Health Organization, 2015.

6. Cao W, Tian W, Hong J, Li D, Tavares R, Noble L, Moss SF and Resnick MB: Expression of bile acid receptor TGR5 in gastric adenocarcinoma. Am J Physiol Gastrointest Liver Physiol 304: G322-G327, 2013.

7. Takahashi M, Nakajima M, Ogata H, Domeki Y, Ohtsuka K, Ihara K, Kurayama E, Yamaguchi S, Sasaki K, Miyachi K and Kato $\mathrm{H}$ : $\mathrm{Cd} 24$ expression is associated with progression of gastric cancer. Hepatogastroenterology 60: 653-658, 2013.

8. Jiang Y, He Y, Li H, Li HN, Zhang L, Hu W, Sun YM, Chen FL and Jin XM: Expressions of putative cancer stem cell markers $\mathrm{ABCB} 1, \mathrm{ABCG} 2$, and CD133 are correlated with the degree of differentiation of gastric cancer. Gastric cancer 15: 440-450, 2012.

9. Yk W, Cf G, T Y, Z C, Xw Z, Xx L, Nl M and Wz Z: Assessment of ERBB2 and EGFR gene amplification and protein expression in gastric carcinoma by immunohistochemistry and fluorescence in situ hybridization. Mol Cytogenet 4: 14, 2011.

10. Hayashi M, Inokuchi M, Takagi Y, Yamada H, Kojima K, Kumagai J, Kawano T and Sugihara K: High expression of HER3 is associated with a decreased survival in gastric cancer. Clin Cancer Res 14: 7843-7849, 2008.

11. Suh JH, Won KY, Kim GY, Bae GE, Lim SJ, Sung JY, Park YK, Kim YW and Lee J: Expression of tumoral FOXP3 in gastric adenocarcinoma is associated with favorable clinicopathological variables and related with hippo pathway. Int J Clin Exp Pathol 8: 14608-14618, 2015.

12. Zhao J, Bai Z, Feng F, Song E, Du F, Zhao J, Shen G, Ji F, Li G and Ma X: Cross-talk between EPAS-1/HIF- $2 \alpha$ and PXR signaling pathway regulates multi-drug resistance of stomach cancer cell. Int J Biochem Cell Biol 72: 73-88, 2016.

13. Kitagawa M, Lee SH and McCormick F: Skp2 suppresses p53-dependent apoptosis by inhibiting p300. Mol Cell 29: 217-231, 2008.

14. Wang Z, Fukushima H, Inuzuka H, Wan L, Liu P, Gao D, Sarkar FH and Wei W: Skp2 is a promising therapeutic target in breast cancer. Front Oncol 1: pii: 18702, 2012.

15. Wei Z, Jiang X, Qiao H, Zhai B, Zhang L, Zhang Q, Wu Y, Jiang H and Sun X: STAT3 interacts with skp2/p27/p21 pathway to regulate the motility and invasion of gastric cancer cells. Cell Signal 25: 931-938, 2013.

16. Cheadle C, Vawter MP, Freed WJ and Becker KG: Analysis of microarray data using z score transformation. J Mol Diagn 5: 73-81, 2003.

17. Smyth GK. Limma: Linear models for microarray data. In: Bioinformatics and Computational Biology Solutions using R and Bioconductor. Gentleman, V. Carey, S. Dudoit, R. Irizarry, W. Huber (eds.). Springer, New York, pp 397-420, 2005.

18. Doncheva NT, Assenov Y, Domingues FS and Albrecht M: Topological analysis and interactive visualization of biological networks and protein structures. Nat Protoc 7: 670-685, 2012.

19. Dennis G Jr, Sherman BT, Hosack DA, Yang J, Gao W, Lane HC and Lempicki RA: David: Database for annotation, visualization, and integrated discovery. Genome biol 4: P3, 2003.

20. Mao X, Cai T, Olyarchuk JG and Wei L: Automated genome annotation and pathway identification using the KEGG orthology (KO) as a controlled vocabulary. Bioinformatics 21: 3787-3793, 2005.

21. de Hoon MJ, Imoto S, Nolan J and Miyano S: Open source clustering software. Bioinformatics 20: 1453-1454, 2004.

22. Singh MS and Michael M: Role of xenobiotic metabolic enzymes in cancer epidemiology. Methods Mol Biol 472: 243-264, 2009.

23. Ding $X$ and Kaminsky LS: Human extrahepatic cytochromes p450: Function in xenobiotic metabolism and tissue-selective chemical toxicity in the respiratory and gastrointestinal tracts. Annu Rev Pharmacol Toxicol 43: 149-173, 2003.

24. González CA, Sala N and Capellá G: Genetic susceptibility and gastric cancer risk. Int J Cancer 100: 249-260, 2002.

25. Shah MA, Khanin R, Tang L, Janjigian YY, Klimstra DS, Gerdes H and Kelsen DP: Molecular classification of gastric cancer: A new paradigm. Clin Cancer Res 17: 2693-2701, 2011.

26. Jelski W and Szmitkowski M: Alcohol dehydrogenase (ADH) and aldehyde dehydrogenase (ALDH) in the cancer diseases. Clin Chim Acta 395: 1-5, 2008. 
27. Duell EJ, Sala N, Travier N, Muñoz X, Boutron-Ruault MC, Clavel-Chapelon F, Barricarte A, Arriola L, Navarro C and Sánchez-Cantalejo E, et al: Genetic variation in alcohol dehydrogenase (ADH1A, ADH1B, ADH1C, ADH7) and aldehyde dehydrogenase (ALDH2), alcohol consumption and gastric cancer risk in the European prospective investigation into cancer and nutrition (EPIC) cohort. Carcinogenesis 33: 361-367, 2012.

28. Saadat M: Genetic polymorphisms of glutathione s-transferase T1 (GSTT1) and susceptibility to gastric cancer: A meta-analysis. Cancer Sci 97: 505-509, 2006.

29. Babhadiashar N, Sotoudeh M, Azizi E, Bashiri J, Didevar R, Malekzadeh R and Ghahremani MH: Correlation between cigarette smoking and urine cotinine level in Gastric cancer patients. Iran J Pharm Res 13: 313-318, 2014.

30. Rodriguez-Antona $C$ and Ingelman-Sundberg M: Cytochrome P450 pharmacogenetics and cancer. Oncogene 25: 1679-1691, 2006.

31. Hurley TD, Edenberg HJ and Li TK: The pharmacogenomics of alcoholism. In: Pharmacogenomics: The Search for Individualized Therapies. Wiley-VCH, Weinheim, pp 417-441, 2002.

32. Vaglenova J, Martínez SE, Portí S, Duester G, Farrés J and Parés X: Expression, localization and potential physiological significance of alcohol dehydrogenase in the gastrointestinal tract. Eur J Biochem 270: 2652-2662, 2003.

33. Jairam S and Edenberg HJ: Single-nucleotide polymorphisms interact to affect adh7 transcription. Alcohol Clin Exp Res 38 921-929, 2014

34. Jelski W,Kutylowska E,Laniewska-Dunaj M and Szmitkowski M: Alcohol dehydrogenase (ADH) and aldehyde dehydrogenase (ALDH) as candidates for tumor markers in patients with pancreatic cancer. J Gastrointestin Liver Dis 20: 255-259, 2011.
35. Yokoyama A and Omori T: Genetic polymorphisms of alcohol and aldehyde dehydrogenases and risk for esophageal and head and neck cancers. Jpn J Clin Oncol 33: 111-121, 2003.

36. Tsukita S and Furuse M: Pores in the wall: Claudins constitute tight junction strands containing aqueous pores. J Cell Biol 149: 13-16, 2000.

37. Hoevel T, Macek R, Swisshelm K and Kubbies M: Reexpression of the TJ protein CLDN1 induces apoptosis in breast tumor spheroids. Int J Cancer 108: 374-383, 2004.

38. Ueda J, Semba S, Chiba H, Sawada N, Seo Y, Kasuga M and Yokozaki H: Heterogeneous expression of claudin-4 in human colorectal cancer: Decreased claudin-4 expression at the invasive front correlates cancer invasion and metastasis. Pathobiology 74: 32-41, 2007.

39. Satake S, Semba S, Matsuda Y, Usami Y, Chiba H, Sawada N, Kasuga $\mathrm{M}$ and Yokozaki $\mathrm{H}$ : $\mathrm{Cdx} 2$ transcription factor regulates claudin-3 and claudin-4 expression during intestinal differentiation of gastric carcinoma. Pathol Int 58: 156-163, 2008.

40. Hewitt KJ, Agarwal R and Morin PJ: The claudin gene family: Expression in normal and neoplastic tissues. BMC Cancer 6: 186, 2006.

41. Matsuda Y, Semba S, Ueda J, Fuku T, Hasuo T, Chiba H, Sawada N, Kuroda Y and Yokozaki H: Gastric and intestinal claudin expression at the invasive front of gastric carcinoma. Cancer Sci 98: 1014-1019, 2007.

42. Choi YL, Kim J, Kwon MJ, Choi JS, Kim TJ, Bae DS, Koh SS, In YH, Park YW, Kim SH, et al: Expression profile of tight junction protein claudin 3 and claudin 4 in ovarian serous adenocarcinoma with prognostic correlation 22: 1185-1195, 2007.

43. Jung H, Jun KH, Jung JH, Chin HM and Park WB: The expression of claudin-1, claudin-2, claudin-3, and claudin-4 in gastric cancer tissue. J Surg Res 167: e185-e191, 2011. 